\title{
Effect of voids in a heat-flux dependent theory for thermoelastic bodies with dipolar structure
}

\section{MARIN MARIN, ANDREAS ÖCHSNER and SORIN VLASE}

\begin{abstract}
.
In our paper we formulate a theory for thermoelastic porous dipolar bodies in which we consider a new independent variable, namely the heat-flux vector. Furthermore, we add, to the differential equations that describe the behavior of the body, a new differential equation which is an equation of evolution which is satisfied by the components of the heat-flux vector. The basic system of the mixed initial-boundary value problem in this context consists of equations of the hyperbolic type. In order to ensure the consistency of the constructed theory, we formulate and prove an uniqueness result, with regards to the solution of the mixed problem.
\end{abstract}

Acknowledgements. Authors are grateful to the referees for several helpful suggestions which contributed to the improvement of the manuscript.

\section{REFERENCES}

[1] Abbas, I. and Marin, M., Analytical solution of thermoelastic interaction in a half-space by pulsed laser heating, Physica E Low Dimens. Syst. Nanostruct., 87 (2017), 254-260

[2] Abd-Elaziz, E. M., Marin, M., and Othman, M. I. A., On the effect of Thomson and initial stress in a thermoporous elastic solid under GN electromagnetic theory, Symmetry-Basel, 11 (2019), No. 3, Art. No. 413

[3] Alamri, S. Z., Khan, A. A., Azeez, M. and Ellahi, R., Effects of mass transfer on MHD second grade fluid towards stretching cylinder: A novel perspective of Cattaneo-Christov heat flux model, Physics Letters A, 383 (2019), 276--281

[4] Choudhuri, S. K. R., On a thermoelastic three-phase-lag model, J. Thermal Stress, 30 (2007), No. 3, 231-238

[5] Cowin, S. C. and Nunziato, J. W., Linear Elastic Materials with Voids, J. Elasticity, 13 (1983), 125-147

[6] Eringen, A. C., Theory of thermo-microstretch elastic solids, Int. J. Eng. Sci., 28 (1990), 1291-1301

[7] Eringen, A. C., Microcontinuum Field Theories, 1999, Springer-Verlag, New York

[8] Fried, E. and Gurtin, M. E., Thermomechanics of the interface between a body and its environment, Continuum Mechanics and Thermodynamics 19 (2007), No. 5, 253-271

[9] Green, A. E. and Naghdi, P. M., Re-examination of the basic postulates of thermomechanics, Proc. R. Soc. Lond. A, 432 (1991), 1171-1194

[10] Green, A. E. and Naghdi, P. M., On undamped heat wave in elastic solids, J. Thermal Stress 15 (1992), No. 2, 253-264

[11] Green, A. E. and Naghdi, P. M., Thermoelasticity without energy dissipation. J. Elast. 9 (1993), 1-8

[12] Green, A. E. and Rivlin, R. S., Multipolar continuum mechanics, Arch. Rational Mech. Anal., 17 (1964), 113-147

[13] Hayat,T, Saif, RS, Ellahi, R. et al., Numerical study for Darcy-Forchheimer flow due to a curved stretching surface with Cattaneo-Christov heat flux and homogeneous-heterogeneous reactions, Results in Physics, 7 (2017), 2886-2892

Received: 21.10.2019; In revised form: 30.01.2020; Accepted: 06.02.2020

2010 Mathematics Subject Classification. 74A15, 74A60, 74G40, 35A15.

Key words and phrases. thermoelasticity, heat-flux vector, dipolar bodies, voids, hyperbolic equations, uniqueness of solution.

Corresponding author: Marin Marin; m.marin@unitbv.ro 
[14] Iesan, D., A theory of thermoelastic materials with voids, Acta Mechanica, 60 (1984), 67-89

[15] Iesan, D. and Ciarletta, M., Non-Classical Elastic Solids, 1993, Longman Scientific and Technical, Harlow, Essex, UK and John Wiley \& Sons, Inc., New York

[16] Lebon, G., Jou, D. and Casas-Vazquez, J., Understanding nonequilibrium thermodynamics: foundations, applications, frontiers, 2008, Springer, Berlin

[17] Lebon, G., A generalized theory of thermoelasticity, J. Tech. Phys., 23 (1982), 37-46

[18] Lord, H. W. and Shulman, Y., A generalized dynamical theory of thermoelasticity, J. Mech. Phys. Solids 15 (1967), No. 5, 299-307

[19] Marin, M., Ellahi, R. and Chirilă, A., On solutions of Saint-Venant's problem for elastic dipolar bodies with voids, Carpathian J. Math., 33 (2017), No. 2, 219-232

[20] Marin, M., An approach of a heat-flux dependent theory for micropolar porous media, Meccanica, 51 (2016), 1127-1133

[21] Marin, M., The Lagrange identity method in thermoelasticity of bodies with microstructure, Int. J. Eng. Sci., 32 (1994), No. 1, 1229-1240

[22] Marin, M., Chirilă, A. and Othman, M. I. A., An extension of Dafermos's results for bodies with a dipolar structure, Appl. Math. Comput., 361 (2019), 680-688

[23] Marin, M., Baleanu, D. and Vlase, S., Effect of microtemperatures for micropolar thermoelastic bodies, Struct. Eng. Mech., 61 (2017), No. 3, 381-387

[24] Mindlin, R. D., Micro-structure in linear elasticity, Arch. Rational Mech. Anal., 16 (1964), 51-78

[25] Muller, I. and Ruggeri, T., Rational extended thermodynamics, 1998, Springer, New York

[26] Nunziato, J. W. and Cowin, S. C., A nonlinear theory of elastic materials with voids, Arch. Rational Mech. Anal., 72 (1979), 175-201

[27] Othman, M. I. A. and Marin, M., Effect of thermal loading due to laser pulse on thermoe-lastic porous medium under G-N theory, Results Phys., 7 (2017), 3863-3872

[28] Straughan, B., Heat waves, in: Applied Mathematical Sciences, vol. 177, 2011, Springer, New York

TRANSILVANIA UNIVERSITY OF BRAŞOV

FACULTY OF MATHEMATICS AND COMPUTER SCIENCE

29 B-DUL EROILOR, 500036 BRAŞOV, ROMANIA

Email address: m.marin@unitbv.ro

Email address: svlasedunitbv.ro

ESSLINGEN UNIVERSITY OF APPLIED SCIENCES

FACULTY OF MECHANICAL ENGINEERING

73728 ESSLINGEN, GERMANY

Email address: andreas. oechsner@gmail.com 